\title{
All-Fiber Burst Mode Laser System Integrated With OCT for Cataract Surgery
}

\author{
D. K. Kesim ${ }^{1}$, H. Kalaycıoğlu ${ }^{2}$, C. Kerse ${ }^{1}$ and F. Omer Ilday ${ }^{1,2}$ \\ ${ }^{1}$ Department of Electrical and Electronics Engineering, METU Ankara, 06800, Turkey \\ ${ }^{2}$ Department of Physics, Bilkent University, 06800 Ankara, Turkey
}

\begin{abstract}
We demonstrate a burst-mode $\mathrm{Yb}$ all-fiber femtosecond laser system integrated with OCT for cataract surgery and aim to enhance further the procedure with lower collateral tissue damage, cleaner, efficient cuts with compact and robust structure.
\end{abstract}

\section{INTRODUCTION}

Cataract condition is responsible for two thirds of preventable visual impairments afflicting nearly 190 million all over the world [1]. Every year, 19 million cataract surgeries are performed worldwide [2]. Femtosecond laser assisted cataract surgery has entered clinical use in recent years as an efficient and safe alternative to the traditional method. However, this laserassisted surgery is in its infancy period and would benefit from lower pulse energies (to minimize collateral effects), shorter operation durations, and more compact laser systems. Fiber lasers can address these requirements with their robustness, compact size and minimal-alignment requiring structure. Further, burst mode operation [3], where high repetition rate pulses are delivered in packets repeated at a relatively low rate, have been shown to produce efficient ablation with minimal collateral thermal effects [4].

\section{DETAILS OF SURGICAL SYSTEM}

Here, we demonstrate the first fiber based burst-mode femtosecond laser device for cataract surgery. The laser is coupled to an optical coherence tomography (OCT) system with computerized controls (Fig. 1).

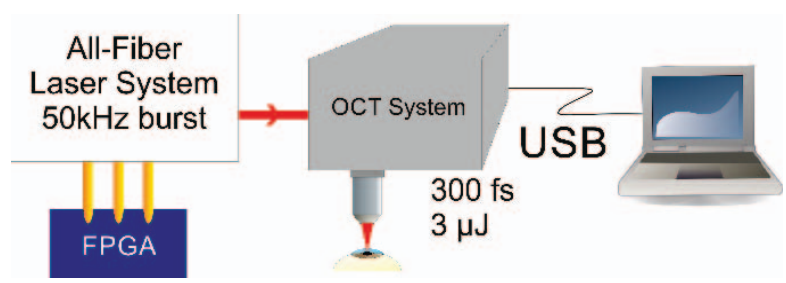

Fig. 1: Laser system integrated with OCT. PC controls scanner and elevator, and gets images from OCT

A home built all fiber $\mathrm{Yb}$ laser amplifier is seeded by a 91$\mathrm{MHz}$ fiber oscillator, followed by a double-clad preamplifier, acusto optic modulator (AOM) pulse picker and a double-clad power amplifier. An FPGA based electronic system triggered by the oscillator is used to impose the desired pulse train on the laser beam. The system is able to produce pulses up to the $5 \mu \mathrm{J}$ range compressible to sub-500 fs. The imaging and tissue processing functions are executed with OCT monitoring, using its galvo scanner with a common beam path. Samples are placed on an elevator controlled by RS232 for vertical scanning. Maximum imaging depth is $1.6 \mathrm{~mm}$ with $8 \mu \mathrm{m}$ axial resolution. The galvo scanner can reach a scan speed of $1 \mathrm{~m} / \mathrm{s}$ with any desired scanning pattern. In our

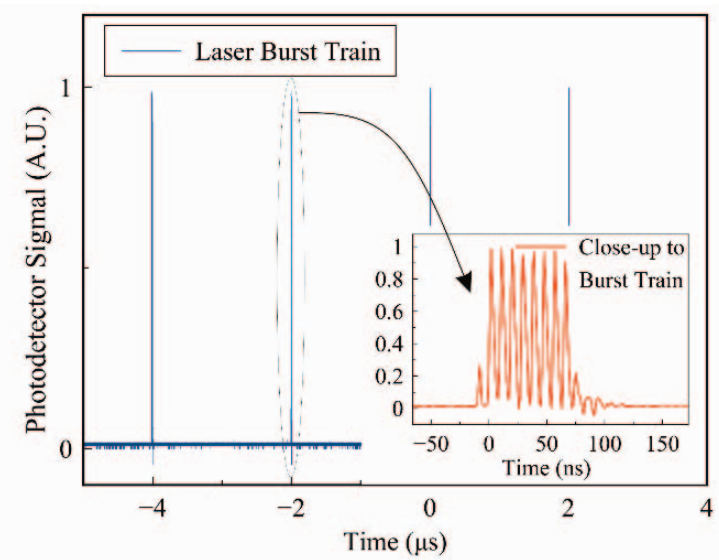

Fig. 2: 8-pulses repeated at $50 \mathrm{kHz}$

preliminary experiments, bursts comprising of 8 pulses with $3 \mu \mathrm{J}$ per pulse at $50 \mathrm{kHz}$ burst repetition rate were applied to agar jells (Fig. 2), which have optical response similar to that of the cornea, as well as cornea obtained from cow eyes. The compressed pulses have $\sim 300$ fs FWHM when the grating compressor is optimized (Fig. 3).

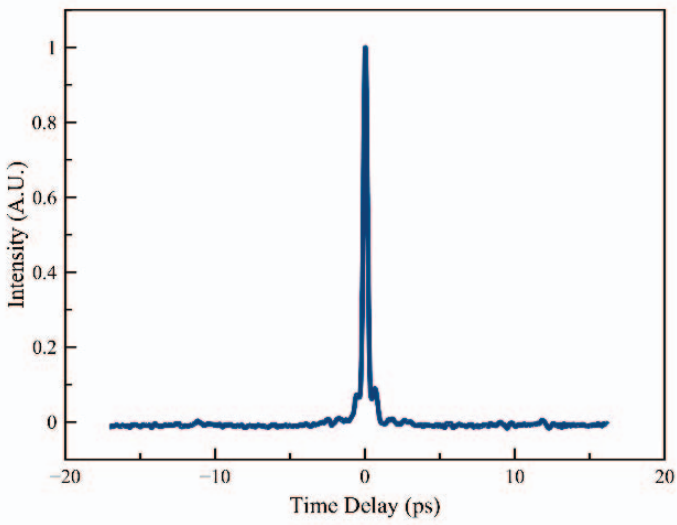

Fig. 3: Autocorrelation of output of laser system. $3 \mu \mathrm{J}$ maximum pulse energies with 300 fs FWHM. 


\section{PRELIMINARY EXPERIMENTS}

Effectiveness of burst mode is demonstrated on agar with bursts with $11.2 \mu \mathrm{J}(1.4 \mu \mathrm{J}$ per pulse energy) while the scan rate was adjusted to obtain $50 \%$ overlap between bursts. For the $400 \mathrm{kHz}$ uniform repetition rate mode scan rate was adjusted to obtain equivalent exposure per spot. The OCT images of the results (Fig. 4(b)) show that while burst mode ablated a substantial volume, uniform repetition rate mode failed to ablate any visible volume (Fig. 5). The valley on figure is due to OCT imaging artifacts (Fig. 4(a)).

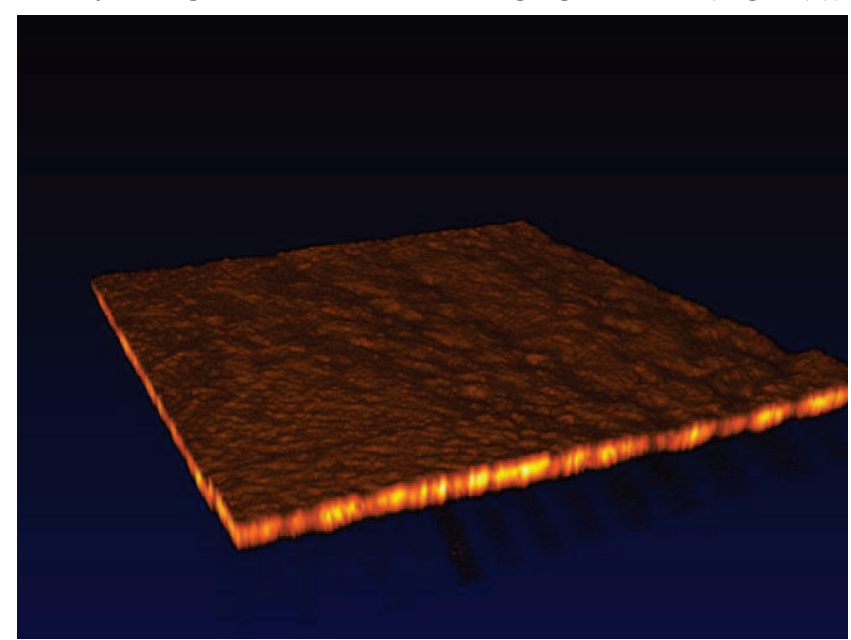

Fig. 4: Agar surface treated with $400 \mathrm{kHz}$ repetition rate pulses of $1.4 \mu \mathrm{J}$ energy each

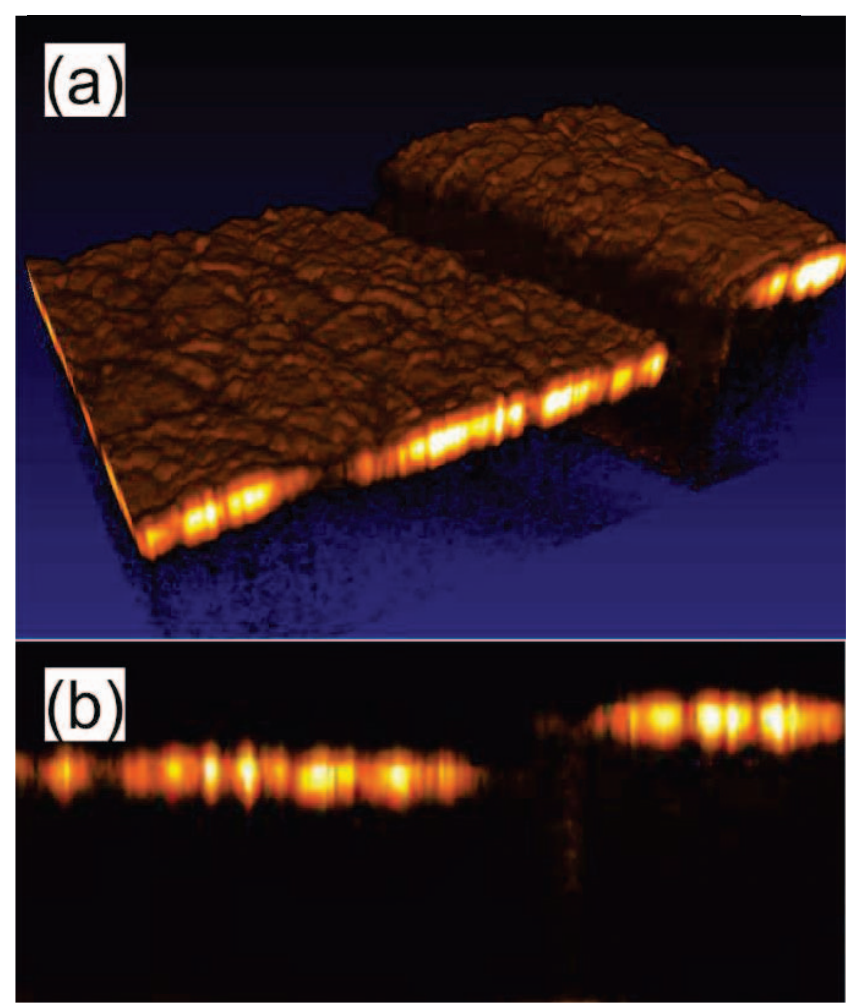

Fig. 5: (a)Agar surface treated with 8 pulse bursts of $1.4 \mu \mathrm{J}$ per pulse energy at a burst repetition rate of $50 \mathrm{kHz}$ (b) Crossection view
Additionally smooth cuts on ex vivo cow cornea by raster scan pattern with $\sim 50 \%$ overlapping spots (Fig 6) were obtained. Agar and ex vivo cow eye trials show that we can create incisions below the surface, in addition to dotted patterns for corneal incision and smooth cuts for capsulorhexis.

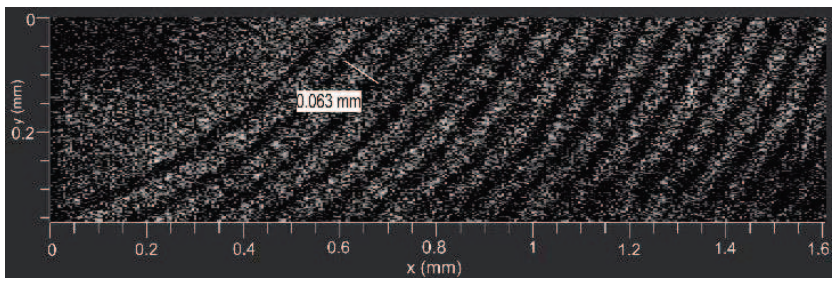

Fig. 6: Raster scanning with 8 pulse bursts containing $1 \mu \mathrm{J}$ pulses on cow cornea.

\section{CONCLUSION}

In summary, a femtosecond burst mode all-fiber $\mathrm{Yb}$ laser amplifier integrated with OCT for cataract surgery is demonstrated for the first time. We show promising results for burst mode with agar and ex vivo experiments on cornea. The system will ultimately be able to create corneal incisions, capsulorhexis and lens fragmentation using a single laser with a common optical path for laser processing and in-situ imaging.

\section{ACKNOWLEDGMENT}

This work was supported by the Scientific and Technological Research Council of Turkey TÜBİTAK under grant no. $112 \mathrm{~T} 980$.

\section{REFERENCES}

[1] World Health Organization,"Universal eye health: a global action plan 2014-2019," WHO Library Cataloguing-in-Publication Data (2013)

[2] K. E. Donaldson et al., "Femtosecond laser assisted cataract surgery," J Caratact Refract Surg, 39, 17531763, (2013)

[3] M. Lapczyna, K.P. Chen, P.R. Herman, H.W. Tan, R.S. Marjoribanks, "Ultra high repetition rate $(133 \mathrm{MHz})$ laser ablation of aluminum with 1.2-ps pulses," Appl. Phys. A 69, S883 (1999).

[4] C. Kerse, H. Kalaycioglu, F. O. Ilday, E. Atalar, "Nonthermal Material and Tissue Processing with $100 \mathrm{MHz}$ and $500 \mathrm{MHz}$ Repetition Rate 